\title{
Dose-volume Histogram Analysis of Knowledge-based Volumetric-modulated Arc Therapy Planning in Postoperative Breast Cancer Irradiation
}

\author{
ERI INOUE ${ }^{1}$, HIROSHI DOI ${ }^{1}$, HAJIME MONZEN $^{2}$, MIKOTO TAMURA $^{2}$, MASAHIRO INADA $^{1}$, \\ KAZUKI ISHIKAWA $^{1}$, KIYOSHI NAKAMATSU ${ }^{1}$ and YASUMASA NISHIMURA ${ }^{1}$ \\ ${ }^{1}$ Department of Radiation Oncology, Kindai University Faculty of Medicine, Osaka, Japan; \\ ${ }^{2}$ Department of Medical Physics, Graduate School of Medical Sciences, Kindai University, Osaka, Japan
}

\begin{abstract}
Background/Aim: We evaluated the dosimetric profiles of manually generated volumetric-modulated arc therapy (VMAT) plans and performance of a commercial knowledge-based planning system (KBP) in treating breast cancer. Materials and Methods: We defined the manually generated VMAT plan as the manual plan (MP). Twenty MPs were generated for left-sided breast cancer patients who underwent breast-conserving surgery and used to develop a KBP training set. The other five patients were used for validation. The dosimetric parameters among MPs, tangential irradiation plans (TPS), and KBP-VMAT plans (KBP-Ps) were compared. Results: $D_{95}$ and homogeneity of the planning target volume (PTV) were significantly higher and greater in MPs and KBP-Ps than in TPs. Lung $V_{20}, V_{40}$. The Dmean for the left anterior descending artery was lower in MPs and KBP-Ps than in TPS. KBP could save time in generating VMAT plans. Conclusion: MPs and KBP-Ps could ensure higher dose uniformity of PTV than TPs. KBP could faster generate comparable MPs for breast cancer.
\end{abstract}

Breast cancer is one of the most common malignancies in women worldwide (1). Partial mastectomy followed by postoperative radiotherapy is a well-established treatment for early-stage breast cancer $(2,3)$. The tangential irradiation technique has been used in postoperative radiotherapy for breast cancer (4).

This article is freely accessible online.

Correspondence to: Eri Inoue, MD, Department of Radiation Oncology, Kindai University Faculty of Medicine, 377-2, Ohnohigashi, Osaka-Sayama, Osaka, Japan. Tel: +81 723660221, Fax: +81 723682388, e-mail: 1876c1@med.kindai.ac.jp

Key Words: Volumetric-modulated arc therapy, intensity-modulated radiation therapy, knowledge-based planning, breast cancer.
However, tangential irradiation for breast cancer presents several issues. Anatomical features such as a pigeon breast might facilitate the delivery of higher doses to the normal lungs (5). It is challenging to generate a treatment plan that facilitates both good conformity of radiation doses administered to the target and reduction in radiation doses administered to the normal organs. Therefore, intensitymodulated radiation therapy (IMRT) has been discussed to have a potential benefit in postoperative radiotherapy for breast cancer (6-12). However, IMRT modalities including volumetric-modulated arc therapy (VMAT) have been less commonly used in clinical practice (9). One of the reasons for this may be that generating IMRT or VMAT plans is more complex and time-consuming than planning for tangential irradiation.

A new commercial knowledge-based planning (KBP) optimization engine, RapidPlan (VarianMedical Systems, Palo Alto, CA, USA), was developed and released for clinical use. RapidPlan predicts achievable dose-volume histograms (DVHs) and automatically generates optimization objectives to realize the prediction. Although the benefits of RapidPlan are still being investigated, there have been many reports of improvements in sparing organs at risk (OARs) using KBP (13-18). The mechanical performance and dosimetric accuracy of KBP have also been verified, showing that KBP could be safely used in clinical practice (17-19). However, to date there are only a few reports on the application of KBP in breast cancer. Thus, this study sought to evaluate the target coverage and normal tissue-sparing profile of conventional tangential plans, manually generated VMAT plans, and VMAT plans generated by KBP in breast cancer.

\section{Materials and Methods}

This study was approved by our institutional review board (approval no. 29-133). 
in vivo $34: 1095-1101(2020)$

Table I. Characteristics of the 20 patients used for a training set and the five patients used for a validation set.

\begin{tabular}{|c|c|c|c|c|}
\hline Characteristics & \multicolumn{2}{|c|}{ Training set $(n=20)$} & \multicolumn{2}{|c|}{ Validation set $(n=5)$} \\
\hline Gender & \multicolumn{2}{|l|}{ All female } & \multicolumn{2}{|l|}{ All female } \\
\hline Age (year) mean (SD) & \multicolumn{2}{|l|}{$63.0(9.7)$} & \multicolumn{2}{|l|}{$54.9(11.4)$} \\
\hline Body mass index mean (SD) & \multicolumn{2}{|l|}{$24.1(4.4)$} & \multicolumn{2}{|l|}{$19.9(2.3)$} \\
\hline \multirow[t]{2}{*}{ Side of breast tumour } & Bilateral & 4 & Bilateral & 1 \\
\hline & Left & 16 & Left & 4 \\
\hline \multirow[t]{8}{*}{ Region of left breast cancer } & $\mathrm{A}$ & 3 & A & 2 \\
\hline & B & 1 & $\mathrm{C}$ & 2 \\
\hline & $\mathrm{C}$ & 4 & $\mathrm{AC}$ & 1 \\
\hline & $\mathrm{D}$ & 2 & & \\
\hline & $\mathrm{E}$ & 1 & & \\
\hline & $\mathrm{AC}$ & 1 & & \\
\hline & $\mathrm{CD}$ & 5 & & \\
\hline & $\mathrm{ACE}$ & 1 & & \\
\hline Smoke (pack years) mean (SD) & $3.9(8.7)$ & & $2.0(4.0)$ & \\
\hline ECOG-PS & 0 & 20 & 0 & 20 \\
\hline \multirow[t]{3}{*}{ Operation technique } & Bp & 4 & Bp & 1 \\
\hline & $\mathrm{Bp}+\mathrm{SNLB}$ & 14 & $\mathrm{Bp}+\mathrm{SNLB}$ & 4 \\
\hline & $\mathrm{Bp}+\mathrm{Ax}(\mathrm{I})$ & 2 & & \\
\hline \multirow[t]{6}{*}{ Pathology of left breast cancer } & Invasive ductal carcinoma & 14 & Invasive ductal carcinoma & 4 \\
\hline & Scirrhous carcinoma & 7 & Scirrhous carcinoma & 2 \\
\hline & Papillotubular carcinoma & 4 & Papillotubular carcinoma & 1 \\
\hline & Solid-tubular carcinoma & 2 & Solid-tubular carcinoma & 1 \\
\hline & Mucinous carcinoma & 1 & Mucinous carcinoma & 0 \\
\hline & Ductal carcinoma in situ & 6 & Ductal carcinoma in situ & 1 \\
\hline \multirow[t]{3}{*}{ Preoperative therapy } & None & 18 & None & 5 \\
\hline & Weekly PTX+Tmab & 1 & & \\
\hline & Weekly PTX+AC & 1 & & \\
\hline \multirow[t]{4}{*}{ Postoperative Stage (UICC 8th ed.) } & 0 & 6 & 0 & 1 \\
\hline & IA & 12 & IA & 2 \\
\hline & IIA & 2 & IB & 1 \\
\hline & & & IIA & 1 \\
\hline Size of tumour $(\mathrm{mm})$, mean $(\mathrm{SD})$ & $9.9(5.7)$ & & $10.8(6.9)$ & \\
\hline \multirow[t]{4}{*}{ Postoperative treatment } & $\mathrm{RT}$ & 7 & $\mathrm{RT}$ & 1 \\
\hline & RT+endocrine therapy & 10 & RT+endocrine therapy & 3 \\
\hline & $\mathrm{RT}+\mathrm{AC}+\mathrm{Tmab}$ & 2 & $\mathrm{RT}+\mathrm{AC}+$ weeklyPTX & 1 \\
\hline & $\mathrm{RT}+\mathrm{Tmab}$ & 1 & & \\
\hline \multirow[t]{2}{*}{ Radiation therapy } & $50 \mathrm{~Gy} / 25 \mathrm{Fr}$ & 17 & $50 \mathrm{~Gy} / 25 \mathrm{Fr}$ & 4 \\
\hline & $60 \mathrm{~Gy} / 30 \mathrm{Fr}$ & 3 & $60 \mathrm{~Gy} / 30 \mathrm{Fr}$ & 1 \\
\hline \multicolumn{5}{|l|}{ Clinical acute events for radiation } \\
\hline \multirow[t]{2}{*}{ Dermatitis } & Grade 1 & 18 & Grade 1 & 4 \\
\hline & Grade 2 & 2 & Grade 2 & 1 \\
\hline Fatigue & Grade 1 & 8 & & \\
\hline \multicolumn{5}{|l|}{ Clinical late events for radiation } \\
\hline \multirow[t]{2}{*}{ Pneumonia } & Grade 1 & 1 & & \\
\hline & Grade 2 & 1 & & \\
\hline Dry skin & Grade 1 & 3 & & \\
\hline Hyperpigmentation & Grade 1 & 1 & Grade 1 & 1 \\
\hline
\end{tabular}

Bp: Partial mastectomy; SNLB: sentinel lymph node biopsy; Ax (I): axillary lymph node (level I) dissection; PTX: paclitaxel; AC: Adriamycin plus cyclophosphamide; Tmab: trastuzumab.

Manually generated VMAT planning and training of KBP. First, 20 VMAT plans were generated in 20 breast cancer patients as a training set for generating KBP. These 20 patients were those who received radiotherapy after breast-conserving surgery for left breast cancer or bilateral breast cancer in 2018 at our hospital. They were treated by tangential irradiation technique and prescribed $50 \mathrm{~Gy}$ in
25 fractions and $10 \mathrm{~Gy}$ in five fractions administered to the tumour bed if positive margins were suspected. Regional lymph nodes were not included in the target area. The characteristics of the included patients are presented in Table I.

Clinical tumour volume (CTV) was defined as the whole breast. For this study, we re-contoured CTV and OARs according to the 

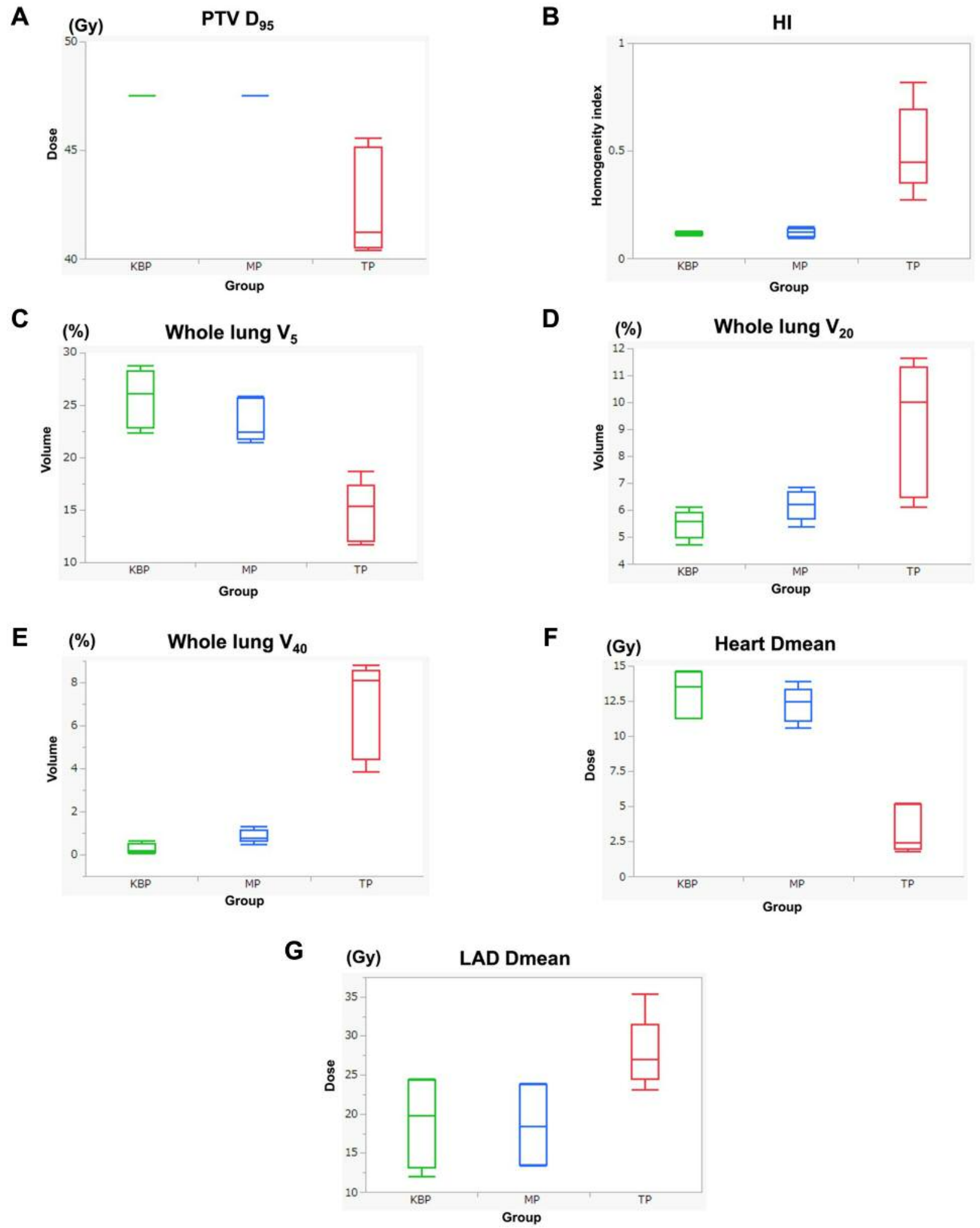

Figure 1. Comparison of dosimetric parameters in each model. (A) Dose irradiated to 95\% volume (D95) for PTV, (B) HI defined as (D2 $D 98) / D 50 \times 100,(C)$ The whole lung volume percentage receiving $5 \mathrm{~Gy}(V 5),(D) V 20$ for the whole lung, (E) V40 for the whole lung, (F) Dmean for the heart, and $(G)$ Dmean for the LAD. TP: Tangent irradiation plan; MP: manually generated plan; KBP: knowledge-based planning PTV: planning target volume; HI: homogeneity index. 
Table II. Dose constraints in OARs for VMAT planning.

\begin{tabular}{lc}
\hline PTV D95 & $\geq 95 \%$ \\
PTV D05 & $\leq 110 \%$ \\
Ipsilateral lung $\mathrm{V}_{20} \mathrm{~Gy}$ & $\leq 33 \%$ \\
Ipsilateral lung $\mathrm{V}_{10} \mathrm{~Gy}$ & $\leq 68 \%$ \\
Ipsilateral lung mean dose & $\leq 20 \%$ \\
Contralateral lung $\mathrm{V}_{20} \mathrm{~Gy}$ & $\leq 8 \%$ \\
Heart $\mathrm{V}_{25} \mathrm{~Gy}$ & $\leq 25 \%$ \\
Heart maximum point dose & $\leq 50 \mathrm{~Gy}$ \\
Contralateral intact breast mean dose & $\leq 5 \mathrm{~Gy}$ \\
Stomach mean dose & $\leq 5 \mathrm{~Gy}$ \\
Cord maximum point dose & $\leq 20 \mathrm{~Gy}$ \\
\hline
\end{tabular}

OARs: Organs at risk; PTV: planning target volume.

Radiation Therapy Oncology Group (RTOG) contouring atlas (20). A margin of $5 \mathrm{~mm}$ was added to the CTV to generate the planning target volume (PTV). For optimization, PTV was limited to within $2 \mathrm{~mm}$ of the body structure. The prescription dose for KBP was a total of 50 Gy in 25 fractions for each patient. Each VMAT plan was designed to cover $95 \%$ of PTV by at least $95 \%$ of the prescription dose (21). The dose constraints for OAR are shown in Table II. We defined the manually generated VMAT plan as the manual plan (MP). MPs included dose constraints to the PTV, contralateral and ipsilateral lungs, heart, right breast, and stomach. Then, the KBP was trained using the 20 MPs.

A commercial treatment planning system (TPS) (Eclipse version 15.6; Varian Medical Systems, Palo Alto, CA, USA), 6-MV photon Flattening Filter Free beams, and four full arcs of VMAT were applied (Gantry angle: $181^{\circ}-179^{\circ}$ clockwise, and $179^{\circ}-181^{\circ}$ counterclockwise) for treatment using Halcyon 1.0 (Varian Medical Systems, Palo Alto, CA, USA). All VMAT plans were optimized with the photon optimizer and calculated with the Varian Analytic Anisotropic Algorithm. In this model, the geometric or dosimetric outliers were not excluded since the removal of statistical outliers had no significant impact on establishing the model (22).

Validation of KBP. The other five patients who received postoperative radiotherapy for left breast cancer were selected for validation sets. The characteristics of these patients are also summarized in Table I. These five patients were also re-contoured according to the RTOG atlas and then five MPs were generated. Five KBP-VMAT plans (KBP-Ps) were generated using KBP. In the optimization process by KBP, optimization objectives termed "line objectives" were placed along the inferior DVH prediction boundary for OARs and priority values were generated by the KBP automatically. For the PTV, upper and lower objectives were used at $50 \mathrm{~Gy}$ and $49.5 \mathrm{~Gy}$, respectively.

Tangential irradiation plans (TPs) were re-planned for recontoured PTV. TPs were planned to be treated by TrueBeam (Varian Medical Systems, Palo Alto, CA, USA) with 6-MV X-rays. The optimal dose distribution was achieved by using a wedge or field-in-field technique.

We compared the dose-volume parameters of each plan using single optimized KBP-Ps, MPs, and TPs. The analysed dosimetric parameters in OARs included the volume receiving $5 \mathrm{~Gy}$ or greater $\left(\mathrm{V}_{5}\right), \mathrm{V}_{20}$, and $\mathrm{V}_{40}$ for lungs; the mean dose (Dmean) for the heart; and the Dmean for the left anterior descending artery (LAD). The dose received by $95 \%$ of the PTV $\left(\mathrm{D}_{95}\right)$ and homogeneity index (HI), defined as $\left(\mathrm{D}_{2}-\mathrm{D}_{98}\right) / \mathrm{D}_{50}$, were also evaluated.

Statistical analysis. Study data are expressed as means with standard deviations in parentheses unless otherwise indicated (Table III). The data were analysed using a matched-paired $t$-test. All analyses were performed using GraphPad Prism version 8.2.1 (GraphPad Software, Inc., San Diego, CA, USA) and differences were considered statistically significant at a $p$-value of less than 0.05 .

\section{Results}

Table III and Figure 1 show the dosimetric results of the TPs, MPs, and KBP-Ps. Figure 2 shows the dose distribution of the TPs, MPs, and KBP-Ps and the DVHs of the MPs and KBP-Ps.

Comparison between MP and TP. The mean $\mathrm{D}_{95}$ values for PTV were 47.5 Gy and 42.6 Gy for the MPs and TPs, respectively; they were significantly different $(p=0.01)$ (Figure 1A). The HI value of MPs was significantly lower than that of TPs $\left(p=0.011\right.$ ) (Figure 1B). Lung $\mathrm{V}_{5}$ values were $23.4 \%$ and $14.6 \%$ for MPs and TPs, respectively; they were significantly different ( $p=0.008$ ) (Figure 1C). Conversely, the lung $\mathrm{V}_{20}$ of MPs was significantly lower than that of TPs $(6.2 \%$ and $9.0 \%$ for MPs and TPs, respectively; $p=0.04$ ) (Figure 1D). Similarly, the lung $\mathrm{V}_{40}$ values were $0.9 \%$ and $6.8 \%$ for MPs and TPs, respectively; they were significantly different $(p=0.003)$ (Figure 1E). Separately, the heart Dmean value of MPs was $12.2 \mathrm{~Gy}$, while that of TPs was $3.3 \mathrm{~Gy}(p=0.0008)$ (Figure 1F).The Dmean for the LAD was significantly lower in MPs than in TPs (27.7 Gy vs. 18.5 Gy; $p=0.024$ ) (Figure 1G).

Comparison between KBP-P and MP. No significant differences in lung $\mathrm{V}_{5}$, heart Dmean, and PTV $\mathrm{D}_{95}$ were observed between KBP-Ps and MPs However, KBP-P achieved slightly but significantly lower lung $\mathrm{V}_{20}$ and $\mathrm{V}_{40}$ in comparison with MPs ( $p=0.0073$ and 0.010 , respectively) (Figure 1D and E). In terms of planning time, it took roughly two hours to generate one MP, while one KBP-P was generated almost within $15 \mathrm{~min}$.

\section{Discussion}

Here, dosimetric parameters of TPs, MPs, and KBP-Ps were compared. MPs and KBP-Ps improved the homogeneity for PTV relative to TPs. However, lung $\mathrm{V}_{5}$ and heart Dmean were increased in VMAT plans, although lung $\mathrm{V}_{20}$ and $\mathrm{V}_{40}$ and LAD Dmean of VMAT plans were significantly lower than those of TPs. These results were similar to those of previous reports comparing dose parameters between TPs and IMRT/VMAT plans (10-12). Increased lung $\mathrm{V}_{5}$ and heart Dmean may constitute disadvantages of VMAT plans in comparison with TPs.

In terms of lung $\mathrm{V}_{5}$, a large-sized clinical trial for locally advanced non-small-cell lung cancer has recently shown that 
Table III. Results of dosimetric parameters for TPs, MPs, and KBP.

\begin{tabular}{|c|c|c|c|c|c|c|}
\hline & $\mathrm{TP}$ & $\begin{array}{c}p \text {-Value } \\
\text { (TP } v s . \mathrm{MP})\end{array}$ & $\begin{array}{c}p \text {-Value } \\
(\mathrm{TP} v s . \mathrm{KBP})\end{array}$ & MP & $\begin{array}{c}p \text {-Value } \\
\text { (MP vs. KBP) }\end{array}$ & KBP \\
\hline \multicolumn{7}{|l|}{ Whole lung } \\
\hline $\mathrm{V}_{5}(\%)$ & $14.6(2.8)$ & 0.0087 & $<0.0001$ & $23.4(2.0)$ & 0.28 & $25.7(2.7)$ \\
\hline $\mathrm{V}_{20}(\%)$ & $9.0(2.5)$ & 0.040 & 0.0025 & $6.2(0.6)$ & 0.0073 & $5.5(0.5)$ \\
\hline $\begin{array}{l}\mathrm{V}_{40}(\%) \\
\text { Heart }\end{array}$ & $6.8(2.5)$ & 0.0030 & 0.0021 & $0.9(0.3)$ & 0.010 & $0.3(0.2)$ \\
\hline Dmean & $3.3(1.6)$ & 0.0008 & 0.0009 & $12.2(1.3)$ & 0.34 & $13.0(1.7)$ \\
\hline $\begin{array}{l}\mathrm{V}_{25}(\%) \\
\mathrm{LAD}\end{array}$ & $3.6(3.1)$ & 0.96 & 0.40 & $3.8(4.2)$ & 0.12 & $6.1(4.9)$ \\
\hline $\begin{array}{l}\text { Dmean (Gy) } \\
\text { PTV }\end{array}$ & $27.7(4.6)$ & 0.024 & 0.033 & $18.5(5.1)$ & 0.43 & $18.9(5.7)$ \\
\hline $\mathrm{D}_{95}(\mathrm{~Gy})$ & $42.5(2.4)$ & 0.010 & 0.010 & $47.5(0.0)$ & Not applicable & $47.5(0.0)$ \\
\hline $\mathrm{HI}$ & $0.5(0.3)$ & 0.011 & 0.011 & $0.1(0.0)$ & 0.51 & $0.1(0.0)$ \\
\hline
\end{tabular}

TP: Tangential irradiation plan; MP: manually generated plan; KBP: knowledge-based planning; PTV: planning target volume; HI: homogeneity index.

lung $\mathrm{V}_{5}$ was not associated with grade 3 radiation pneumonitis (23). In the RTOG0617 trial, lung $\mathrm{V}_{5}$ was $61.6 \%$. Even though lung $\mathrm{V}_{5}$ was increased relative with TPs in this study, its values were only $23.4 \%$ and $25.7 \%$ in MPs and KBP-Ps, respectively. Lung $\mathrm{V}_{20}$ and $\mathrm{V}_{40}$ were significantly lower in VMAT plans than in TPs. These results indicate that VMAT plans were safer for radiation pneumonitis than tangential irradiation.

Radiation delivery to the heart in this context is unavoidable. In the MPs and KBP-Ps, heart Dmean was approximately four times larger than that in TPs. The heart dose is increased in this way because of the optimization method. Here, we constrained the contralateral and ipsilateral lungs' doses strongly, and the beam angles were designed to avoid both lungs. However, those constraints could increase the radiation doses to the mediastinum area including the heart (Figure 2A). Darby et al. have shown that the rate of major coronary events for breast cancer increased linearly by $7.4 \%$ per Gy of mean heart dose (24). However, Abouegylah et al. have revealed that the dose to the LAD was a significant parameter to use to predict the risk of radiationinduced cardiotoxicity (25). In the case of left-sided tangential irradiation, The LAD is close to the PTV and tended to receive a higher dose. Here, the LAD Dmean was significantly lower in VMAT plans than in TPs. The heart Dmean is calculated from the heterogeneous dose distribution including both high- and low-dose radiation areas. Therefore, we considered the LAD Dmean as more important than heart Dmean for discerning late complications of cardiac events, so the MPs and KBP-Ps were acceptable.

Both KBP-P and MP generated similar VMAT plans with no significant differences in $\mathrm{HI}$, lung $\mathrm{V}_{5}$, heart and LAD. Therefore, it can be argued that we could create VMAT plans with almost the same quality using KBP. $\mathrm{KBP}$ tended to suppress lung $\mathrm{V}_{20}$ and $\mathrm{V}_{40}$ doses more significantly than those seen in MPs. Only few studies have described the use of KBP in breast cancer. Some reports have suggested that KBP was useful even if the planner is a beginner $(14,26,27)$. Overall, this study suggests that MPs and KBP-Ps were acceptable developments following breast-conserving surgery, and KBP could generate almost the same VMAT plans as MPs within a short time. Further validation studies should focus on the feasibility of KBPs for breast cancer.

\section{Conclusion}

After breast-conserving surgery, a single optimized KBP could generate VMAT plans comparable to MPs while also saving time.

\section{Conflicts of Interest}

The Authors declare that no conflicts of interest exist regarding this study.

\section{Authors' Contributions}

Concept and design: EI, HD, HM, MT. Treatment planning: EI, HD, MI, KI, KN. Clinical evaluation: EI, HD. Data analysis: EI, HD, HM, MT. Manuscript preparation: EI, HD, HM, MT, YN. All Authors read and approved the final manuscript.

\section{Acknowledgements}

Authors would like to thank Enago (www.enago.jp) for the English language review.

\section{Funding}

The study was partially supported by the National Cancer Center Research and Development Funds (29-A-3), and a Grant JSPS KAKENHI Grant Number JP17K16493, JP19K08135. 

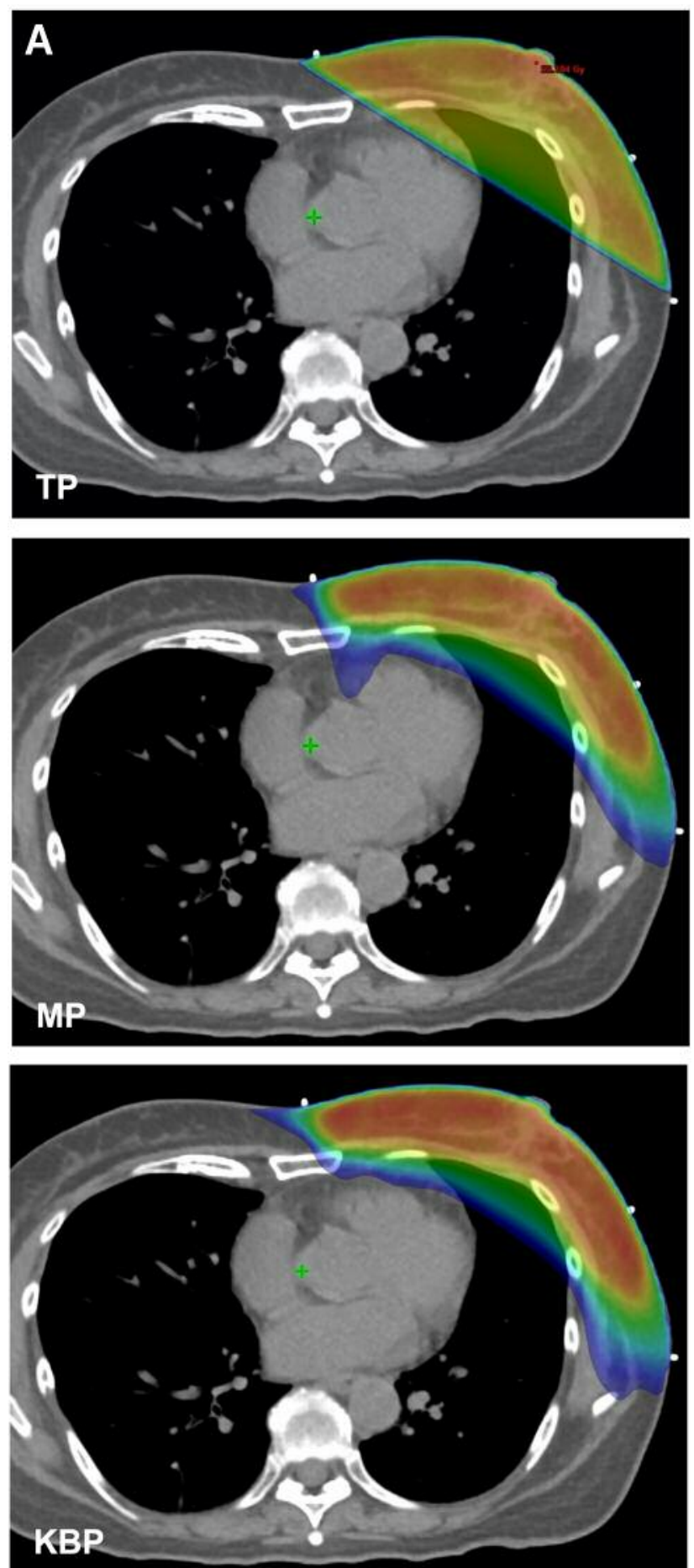

\section{References}

1 Bray F, Ferlay J, Soerjomataram I, Siegel RL, Torre LA and Jemal A: Global cancer statistics 2018: GLOBOCAN estimates of incidence and mortality worldwide for 36 cancers in 185 countries. CA Cancer J Clin 68(6): 394-424, 2018. PMID: 30207593. DOI: $10.3322 /$ caac. 21492

2 Early Breast Cancer Trialists' Collaborative Group: Favourable and unfavourable effects on long-term survival of radiotherapy

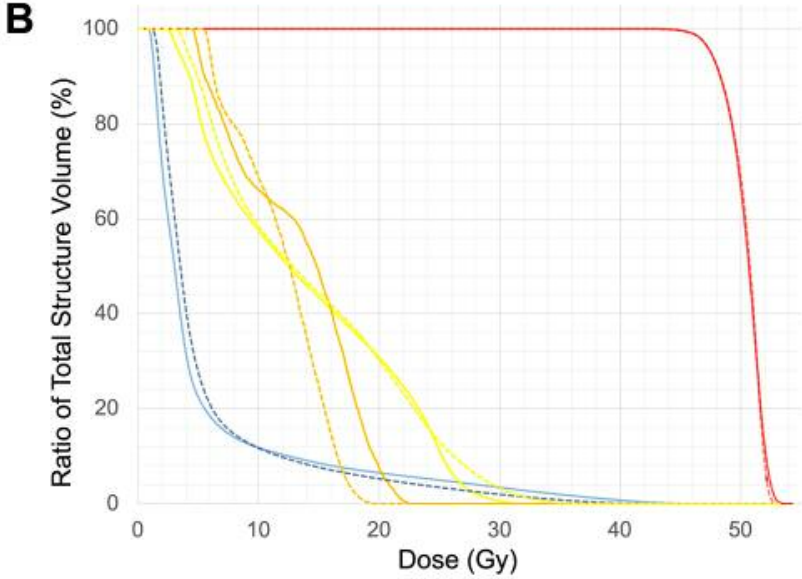

Figure 2. Dose distribution and DVH of a patient with left breast cancer after breast-conserving surgery. (A) Treatment plans with corresponding examples of a tangent irradiation plan (TP), manually generated VMAT plan $(M P)$, and VMAT plan using KBP (KBP). (B) The DVH of VMAT planning. The solid lines are the average of MPs and the dotted lines are the average of KBP-Ps. (red: PTV, orange: LAD, yellow: heart, blue: whole lungs). VMAT: Volumetric-modulated arc therapy; KBP: knowledge-based planning; PTV: planning target volume; LAD: left anterior descending artery.

for early breast cancer: an overview of the randomised trials. Lancet 355: 1757-1770, 2000. DOI: 10.1016/S01406736(00)02263-7

3 Early Breast Cancer Trialists' Collaborative Group (EBCTCG), Darby S, McGale P, Correa C, Taylor C, Arriagada R, Clarke M, Cutter D, Davies C, Ewertz M, Godwin J, Gray R, Pierce L, Whelan T, Wang Y and Peto R: Effect of radiotherapy after breast-conserving surgery on 10-year recurrence and 15-year breast cancer death: meta-analysis of individual patient data for 10,801 women in 17 randomised trials. Lancet 378(9804): 17071716, 2011. PMID: 22019144. DOI: 10.1016/S01406736(11)61629-2

4 MacDonald SM and Napolitano B: Early breast cancer. In: Target volume delineation and field setup. Lee NY, Lu JJ (eds.). Springer-Verlag, Berlin Heidelberg, 2013. DOI: 10.1007/978-3642-28860-9_10. Available at: https://documents.pub/document/ target-volume-delineation-and-field-setup-early-breastcancer.html

5 Das IJ, Cheng EC, Freedman G and Fowble B: Lung and heart dose volume analyses with CT simulator in radiation treatment of breast cancer. Int J Radiat Oncol Biol Phys 42(1): 11-19, 1998. PMID: 9747814. DOI: 10.1016/s0360-3016(98)00200-4

6 Pignol JP, Olivotto I, Rakovitch E, Gardner S, Sixel K, Beckham W, Vu TT, Truong P, Ackerman I and Paszat L: A multicenter randomized trial of breast intensity-modulated radiation therapy to reduce acute radiation dermatitis. J Clin Oncol 26(13): 20852092, 2008. PMID: 18285602. DOI: 10.1200/JCO.2007.15.2488

7 Ho AY, Ballangrud A, Li G, Gupta GP, McCormick B, Gewanter R, Gelblum D, Zinovoy M, Mueller B, Mychalczak B, Dutta P, Borofsky K, Parhar P, Reyngold M, Braunstein LZ, Chawla M, Krause K, Freeman N, Siu CT, Cost Z, Arnold BB, Zhang Z and 
Powell SN: Long-term pulmonary outcomes of a feasibility study of inverse-planned, multibeam intensity modulated radiation therapy in node-positive breast cancer patients receiving regional nodal irradiation. Int J Radiat Oncol Biol Phys 103(5): 1100-1108, 2019. PMID: 30508620. DOI: 10.1016/j.ijrobp.2018.11.045

8 Teoh M, Clark $\mathrm{CH}$, Wood $\mathrm{K}$, Whitaker S and Nisbet A: Volumetric modulated arc therapy: a review of current literature and clinical use in practice. Br J Radiol 84(1007): 967-996, 2011. PMID: 22011829. DOI: $10.1259 / \mathrm{bjr} / 22373346$

9 Balaji K, Subramanian B, Yadav P, Radha CA and Ramasubramanian V: Radiation therapy for breast cancer: literature review. Med Dosim 41(3): 253-257, 2016. PMID: 27545009. DOI: 10.1016/j.meddos.2016.06.005

10 Lohr F, El-Haddad M, Dobler B, Grau R, Wertz HJ, KrausTiefenbacher U, Steil V, Madyan YA and Wenz F: Potential effect of robust and simple IMRT approach for left-sided breast cancer on cardiac mortality. Int J Radiat Oncol Biol Phys 74(1): 73-80, 2009. PMID: 18973977. DOI: 10.1016/j.jirobp.2008.07.018

11 Aras S, İkizceli T and Aktan M: Dosimetric comparison of threedimensional conformal radiotherapy (3D-CRT) and intensity modulated radiotherapy techniques (IMRT) with radiotherapy dose simulations for left-sided mastectomy patients. Eur J Breast Health 15(2): 85-89, 2019. PMID: 31001609. DOI: 10.5152/ ejbh.2019.4619

12 Haciislamoglu E, Colak F, Canyilmaz E, Dirican B, Gurdalli S, Yilmaz AH, Yoney A and Bahat Z: Dosimetric comparison of left-sided whole-breast irradiation with 3DCRT, forward-planned IMRT, inverse-planned IMRT, helical tomotherapy, and volumetric arc therapy. Phys Med 31(4): 360-367, 2015. PMID: 25733372. DOI: 10.1016/j.ejmp.2015.02.005

13 Fogliata A, Nicolini G, Bourgier C, Clivio A, De Rose F, Fenoglietto P, Lobefalo F, Mancosu P, Tomatis S, Vanetti E, Scorsetti M and Cozzi L: Performance of a knowledge-based model for optimization of volumetric modulated arc therapy plans for single and bilateral breast irradiation. PLoS One 10(12): e0145137, 2015. PMID: 26691687. DOI: 10.1371/journal.pone.0145137

14 Wang J, Hu W, Yang Z, Chen X, Wu Z, Yu X, Guo X, Lu S, Li $\mathrm{K}$ and $\mathrm{Yu} \mathrm{G}$ : Is it possible for knowledge-based planning to improve intensity modulated radiation therapy plan quality for planners with different planning experiences in left-sided breast cancer patients? Radiat Oncol 12(1): 85, 2017. PMID: 28532508. DOI: 10.1186/s13014-017-0822-z

15 Fogliata A, Nicolini G, Clivio A, Vanetti E, Laksar S, Tozzi A, Scorsetti M and Cozzi L: A broad scope knowledge-based model for optimization of VMAT in esophageal cancer: validation and assessment of plan quality among different treatment centers. Radiat Oncol 10: 220, 2015. PMID: 26521015. DOI: 10.1186/s13014-0150530-5

16 Kamima T, Ueda Y, Fukunaga JI, Shimizu Y, Tamura M, Ishikawa $\mathrm{K}$ and Monzen H: Multi-institutional evaluation of knowledgebased planning performance of volumetric modulated arc therapy (VMAT) for head and neck cancer. Phys Med 64: 174-181, 2019. PMID: 31515017. DOI: 10.1016/j.ejmp.2019.07.004

17 Ueda Y, Fukunaga JI, Kamima T, Adachi Y, Nakamatsu K and Monzen H: Evaluation of multiple institutions' models for knowledge-based planning of volumetric modulated arc therapy (VMAT) for prostate cancer. Radiat Oncol 13(1): 46, 2018. PMID: 29558940. DOI: 10.1186/s13014-018-0994-1

18 Tamura M, Monzen H, Matsumoto K, Kubo K, Otsuka M, Inada M, Doi H, Ishikawa K, Nakamatsu K, Sumida I, Mizuno H,
Yoon DK and Nishimura Y: Mechanical performance of a commercial knowledge-based VMAT planning for prostate cancer. Radiat Oncol 13(1): 163, 2018. PMID: 30170614. DOI: 10.1186/s13014-018-1114-y

19 Kubo K, Monzen H, Ishii K, Tamura M, Kawamorita R, Sumida I, Mizuno $\mathrm{H}$ and Nishimura $\mathrm{Y}$ : Dosimetric comparison of RapidPlan and manually optimized plans in volumetric modulated arc therapy for prostate cancer. Phys Med 44: 199-204, 2017. PMID: 28705507. DOI: 10.1016/j.ejmp.2017.06.026

20 Breast cancer contouring atlas. Available at: https://www.rtog.org/ CoreLab/ContouringAtlases/BreastCancerAtlas.aspx [Last accessed 05/02/2020]

21 Das IJ, Cheng CW, Chopra KL, Mitra RK, Srivastava SP and Glatstein E: Intensity-modulated radiation therapy dose prescription, recording, and delivery: patterns of variability among institutions and treatment planning systems. J Natl Cancer Inst 100(5): 300-307, 2008. PMID: 18314476. DOI: 10.1093/jnci/djn020

22 Hussein M, South CP, Barry MA, Adams EJ, Jordan TJ, Stewart AJ and Nisbet A: Clinical validation and benchmarking of knowledge-based IMRT and VMAT treatment planning in pelvic anatomy. Radiother Oncol 120(3): 473-479, 2016. PMID: 27427380. DOI: $10.1016 /$ j.radonc.2016.06.022

23 Chun SG, Hu C, Choy H, Komaki RU, Timmerman RD, Schild SE, Bogart JA, Dobelbower MC, Bosch W, Galvin JM, Kavadi VS, Narayan S, Iyengar P, Robinson CG, Wynn RB, Raben A, Augspurger ME, MacRae RM, Paulus R and Bradley JD: Impact of intensity-modulated radiation therapy technique for locally advanced non-small-cell lung cancer: A secondary analysis of the NRG oncology RTOG 0617 randomized clinical trial. J Clin Oncol 35(1): 56-62, 2017. PMID: 28034064. DOI: 10.1200/ JCO.2016.69.1378

24 Darby SC, Ewertz M, McGale P, Bennet AM, Blom-Goldman U, Brønnum D, Correa C, Cutter D, Gagliardi G, Gigante B, Jensen MB, Nisbet A, Peto R, Rahimi K, Taylor C and Hall P: Risk of ischemic heart disease in women after radiotherapy for breast cancer. N Engl J Med 368(11): 987-998, 2013. PMID: 23484825. DOI: 10.1056/NEJMoa1209825

25 Abouegylah M, Braunstein LZ, Alm El-Din MA, Niemierko A, Salama L, Elebrashi M, Edgington SK, Remillard K, Napolitano B, Naoum GE, Sayegh HE, Gillespie T, Farouk M, Ismail AA and Taghian AG: Evaluation of radiation-induced cardiac toxicity in breast cancer patients treated with trastuzumab-based chemotherapy. Breast Cancer Res Treat 174(1): 179-185, 2019. PMID: 30478787. DOI: 10.1007/s10549-018-5053-y

26 Kubo K, Monzen H, Ishii K, Tamura M, Nakasaka Y, Kusawake M, Kishimoto S, Nakahara R, Matsuda S, Nakajima $T$ and Kawamorita R: Inter-planner variation in treatment-plan quality of plans created with a knowledge-based treatment planning system. Phys Med 67:132-140, 2019. PMID: 31706149. DOI: 10.1016/j.ejmp.2019.10.032

27 Uehara T, Monzen $\mathrm{H}$, Tamura $\mathrm{M}$, Ishikawa $\mathrm{K}$, Doi $\mathrm{H}$ and Nishimura Y: Dose-volume histogram analysis and clinical evaluation of knowledge-based plans with manual objective constraints for pharyngeal cancer. J Radiat Res (in press)

Received January 22, 2020

Revised February 4, 2020

Accepted February 10, 2020 